\title{
Laboreal
}

Volume $14 \mathrm{~N}^{\circ} 2$ | 2018

Digitalização e evolução do trabalho real

\section{Digitalização e evolução do trabalho real: introdução}

Digitalización y evolución del trabajo real: introducción

Digitalisation et évolution du travail réel: introduction

Digitalization and the evolution of real work: introduction

\section{Carole Baudin e Patricio Nusshold}

\section{CpenEdition}

Journals

Edição electrónica

URL: http://journals.openedition.org/laboreal/582

DOI: $10.4000 /$ laboreal.582

ISSN: 1646-5237

\section{Editora}

Universidade do Porto

Refêrencia eletrónica

Carole Baudin e Patricio Nusshold, « Digitalização e evolução do trabalho real: introdução », Laboreal [Online], Volume 14 №2 | 2018, posto online no dia 01 dezembro 2018, consultado o 24 setembro 2020. URL : http://journals.openedition.org/laboreal/582 ; DOI : https://doi.org/10.4000/laboreal.582

Este documento foi criado de forma automática no dia 24 setembro 2020.

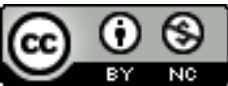

Laboreal está licenciado com uma Licença Creative Commons - Atribuição-NãoComercial 4.0 Internacional. 


\title{
Digitalização e evolução do trabalho real: introdução
}

\author{
Digitalización y evolución del trabajo real: introducción \\ Digitalisation et évolution du travail réel: introduction \\ Digitalization and the evolution of real work: introduction
}

\section{Carole Baudin e Patricio Nusshold}

\section{NOTA DO EDITOR}

http://dx.doi.org/10.15667/laborealxiv218cbpnpt

1 Ao elaborar a solicitação de artigos para deste dossier temático, iniciou-se um debate entre os membros do comitê: deveríamos falar-se de digitalização ou númerização?

De fato os franceses, reticentes em relação à apropriação de palavras inglesas, quase não utilizam a palavra 'digitalização', que é utilizada noutros países francófonicos, hispânicos e lusófonos. Mas para além do debate linguístico e cultural, o uso (ou não) deste termo é revelador de problemas paradigmáticos.

O primeiro problema tem a ver com a confusão do seu significado. Como apresenta A. Mathé (2015), em francês, a diferença semântica reside em um destaque para o numérico (em oposição ao analógico) para designar os suportes da revolução tecnológica, em sua materialidade, enquanto o digital e a digitalização seriam termos mais usados para descrever as experiências que provocariam essas materialidades. Mesmo que nestes discursos a diferença não seja tão clara, esta primeira reflexão mostra a complexidade da análise que, ao assimilar este fenómeno tecnológico em termos de causas, meios e consequências, acaba por definir a abordagem com a qual olhamos para fenômeno digital/numérico.

O segundo problema é que, muitas vezes o termo 'digitalização' é usado para instaurar uma ruptura na revolução tecnológica. Anuncia-se uma 'revolução' (de fato, muitos fazem referência à uma quarta revolução industrial). Do ponto de vista histórico e 
antropológico (podemos citar os trabalhos de Leroi-Gourhan, Mauss, Simondon, Lemonnier, Gras), a digitalização faz parte de uma dinâmica evolutiva e contínua das técnicas criadas pelo homem. Entretanto a dinâmica tecnológica vivida atualmente, cujo ponto de inflexão se iniciou com a chegada das Tecnologias da Informação e Comunicação (TIC), está traçando uma trajetória exponencial tanto em sua magnitude como sua rapidez. De fato, alguns autores mostram o contínuo e o disrruptivo no processo do fenômeno digital (Valenduc \& Vendramin, 2016), ilustrando a complexidade da leitura histórica.

Na literatura, a digitalização tem sido considerada sob diversas formas (automatização/ robotização da produção, surgimento da economia colaborativa, surgimento da mão de obra digital, trabalho à pedido através de aplicações) e seus efeitos têm sido analisados a partir de várias perspectivas: 1) através de pesquisas frequentemente citadas, como Frey \& Osborne (2017), e o estudo da Organização para a Cooperação e o Desenvolvimento Econômico (OCDE, 2017) sobre os riscos da automatização de profissões e empregos. No mesmo sentido, vários trabalhos têm estudado os impactos da economia digital no mercado laboral, no emprego e na proteção social (Degryse, 2016, 2017; Drahokoupil \& Fabo, 2016; Murillo, Buckland, \& Val, 2017; Valenduc \& Vendramin, 2016, 2017; Watson \& Stafford, 2016). 2) A reviravolta digital também é estudada a partir de seu impacto nas formas de organização, gestão e liderança nas empresas (Loebbecke \& Picot, 2015). Paralelamente, a natureza, a estrutura e o valor das atividades laborais e as relações sociais e individuais relacionada com elas (tecnoestresse, hiperconectividade, hiperflexibilidade, precariedade social, fronteiras esbatidas entre a vida e o trabalho) têm emergido como temas centrais na literatura (Ayyagari, Grover \& Purvis, 2011; De Stefano, 2015; Shu, Tu \& Wang, 2011). 3) Finalmente, as transformações digitiais são percebidas como desafios do ponto de vista legal, da legislação laboral (Prassl \& Risak, 2016), da proteção laboral ou da gestão de fluxos de dados.

6 Este resumo bibliográfico mostra que estamos no início de uma mudança estrutural drástica no mundo do trabalho. Não obstante, poucos são os estudos que relatam as mudanças efetivas provocadas nas práticas reais de trabalho. Mesmo que desde o fim dos anos 90 se tenha produzido muito conhecimento sobre a introdução das TICs no trabalho, seus efeitos nas formas de trabalho, de interação, de construir novas práticas, entre outros temas, o fenômeno que estamos vivendo vai além dos sintomas observados com a introdução de computadores ou certas aplicações informáticas no trabalho.

7 Se trata de algo mais 'proteiforme' (Mériau \& Rousseau, 2017), visto que ao abarcar transformações a níveis e registros distintos do trabalho, provoca efeitos multidimensionais e interdependentes (complexos) que não são bem conhecidos / e descritos. A comunidade científica e especializada nas condições de trabalho está iniciando um trabalho de recompilação de experiências [ $\left.{ }^{[}\right]$, gerando assim um corpus de conhecimento no qual se enquadra o presente número.

8 Neste enquadramento, a proposta deste dossier temático é, através da consideração de estudos realizados em distintas regiões e setores, 1) observar como se aprende a dinâmica digital em contextos distintos; 2) para contribuir na produção de conhecimento baseado na compreensão de seu impacto nas experiências laborais; e 3) para colocar em debate os métodos de análise e intervenção que permitem otimizar as 'novas' condições de trabalho. 


\section{Focalização no fenômeno digital}

primeiro artigo, de Deli Salini, Juana Sarmiento Jaramillo, Annie Goudeaux y Germain Poizat apresenta dois estudos feitos na Suíça sobre a implementação do que nomeiam 'Objetos Técnicos Digitais (OTD)' no processo de documentação de informação sobre utilizadores em duas profissões de prestação de serviços: enfermaria e escritórios de oficiais de justiça. Esta contribuição analisa as implicações da difusão destes OTDs nas práticas quotidianas dos trabalhadores envolvidos, assim como suas percepções dos impactos com a finalidade de construir uma intervenção de formação original.

11 O segundo artigo de Sylvain Leduc y Ludovic Ponge traz o tema da 'Evolução Digital' (ED), implementada em dois grandes grupos de serviços privados franceses na área de transportes aéreos e banca. Em suas análises ao autores mostram que esta ED abarca novos sistemas numéricos, mas também novas formas de organização do trabalho. Através de uma análise sobre as modificações provocadas no sistema de trabalho, os autores propõem uma reflexão sobre a prática de intervenção ergonômica que implica essas transformações.

$12 \mathrm{O}$ terceiro artigo de Elodie Chambonnière, Jacqueline Vacherand-Revel y Bruno Andrieu apresenta os primeiros resultados de um trabalho de doutoramento que analisa a transformação da atividade laboral de uma encarregada de um grande grupo francês da construção civil, com a integração de um dispositivo informático que tem como suporte um tablet táctil. Mas para além do resultado, esta contribuição propõe uma reflexão sobre os quadros teóricos convocados para observar e analisar as implicações do fenômeno digital nas dimensões situadas e mediatizadas das atividades laborais desta atividade produtiva.

13 Finalmente o quarto artigo de Sacha Pinheiro, Marta Santos y Liliana Cunha descreve as condições de implementação e os impactos do projeto de digitalização desenvolvido no Instituto de Segurança Social, no Brasil. Este amplo estudo ao nível de escala e tempo, permite às autoras tirar conclusões sobre as condições de implementação e seus impactos sobre as formas de apropriação das mudanças.

14 Em todos os casos descritos, a digitalização consiste na integração de suportes técnicos cujo objetivo é otimizar o tratamento, a gestão (análise, resolução) e a difusão de informações. Cabe destacar que todos os autores mostram que estes novos suportes de sustentam em transformações organizacionais modificando os fluxos de trabalho e os objetivos de produtividade. Parece-no importante sinalizar que estas evoluções não representam uma redução do trabalho humano, pelo contrário, uma transformação da atividade, indispensável para poder encontrar soluções criativas aos problemas para os quais a tecnologia é insuficiente.

15 A digitalização descrita não é somente implementação de sistemas especializados. Tratase da implementação de dispositivos multi-suportes, multi-modais, automáticos e artificialmente inteligentes, tratando e gerando a multiplicidade de dados, controlados por algoritmos, categorizando e interpretando fenômenos complexos para a tomada de decisões e a intervenção em distintos níveis da esfera do trabalho.

16 Para entender as consequências e novas práticas laborais, os autores do presente dossier focam o fenômeno digital no trabalho a partir de um prisma compreensivo, vendo-o numa perspectiva de construção social de um ponto de vista 
antropotecnológico (Salini et al.) ou com um enfoque instrumental da análise da atividade mediatizada (Leduc \& Ponge, Chambonnière et al., Pinheiro et al.), inspirado nos trabalhos de autores como Rabardel (1995) para analisar as transformações na interação com os artefatos de trabalho, sob um enfoque da 'clínica do uso' (BobillierChaumon \& Clot, 2016). Assim, os objetos digitais do trabalho são vistos não somente como uma contingência física no trabalho, mas como uma contingência sócio-técnica, condição necessária para entender o papel dos novos não-humanos no trabalho (Latour, 1991).

\section{Contribuições para um corpus teórico: impactos e novas práticas}

Muito embora a digitalização tome formas distintas, existe uma configuração frequentemente encontrada nos casos descritos em que os dispositivos digitais são designados como novas instâncias de inteligibilidade (Sadin, 2015) e de corporeidade (Baudin, 2017a) no trabalho real. Novos dispositivos em relação aos quais os trabalhadores têm pouca margem de ação e apropriação.

Assim, as quatro contribuições expõem de que maneira os objetos digitais estão modificando as práticas laborais em 1) sua materialidade espacial, temporal e interacional; 2) sua organização individual, coletiva, social e funcional; e 3) suas representações individuais e coletivas.

191 - Ao propor novos suportes virtuais, móveis e multimodais, para acompanhar a atividade, geram-se novas exigências e cargas para os sujeitos e os coletivos de trabalho. Por exemplo, Leduc e Ponge mostram como a integração de suportes móveis na atividade dos operadores de aeroporto provoca um aumento de variabilidade dos objetivos e dos lugares de trabalho, obrigando as pessoas a atuar de forma permanente em contextos dinâmicos e instáveis. Salini et al., assim como Pinheiro et al., evidenciam as mudanças de raciocínio gerados pelo suporte numérico no trabalho em escritório. A apresentação de informações de tipos diversos, necessária para tratar um problema, concentra-se sobre um (ou dois) ecrã (s) que apresenta as informações necessárias para a resolução, de forma linear e sequencial, obrigando os operadores a realizar manipulações permanentes para ver um ou outro documento eletrônico, enquanto antes os documentos impressos possibilitavam dispor a informação em forma global, heurística e imediata. Sem falar das solicitações gerados ao nível de carga visual e postural. Chambonnière et al. identificam as vantagens em poder apresentar informações em suportes móveis para o trabalho da direção de obras, mas assinalam também como impedem ou atrapalham atividades mais informais que normalmente são executadas para compensar atrasos. Enfim, todos os casos que analisam atividades de serviços revelam as transformações que estes novos suportes operam na interação com os clientes seja porque enviam uma imagem de maior eficiência, elevando assim o nível de exigência dos cliente, ou porque se opõem às representações que os clientes têm da atividade (como o caso das enfermeiras, Salini et al.).

2 - Ao implementar sistemas informáticos codificados e interconectados, os trabalhadores vêem-se na obrigação de se adaptar às novas lógicas de categorização de suas atividades, gerando novas cargas cognitivas, e solicitando novas competências individuais e coletivas. Assim, Salini et al., mostram em que medida as enfermeiras se questionam sobre as formas de assegurar a nova evolução clínica do paciente ou 
'dossier cliente', sobre o fornecimento de detalhes, quem será o destinatário, etc. Entra em jogo também o estatuto de 'saber profano' (Baudin, 2017b), este saber que os operadores constroem ao longo da experiência laboral. Um saber que pertence ao registro do sentido, do percebido, o que não tem lugar no programa informático. Chambonnière et al., entretanto, descrevem pouco o sistema em si, fazem alusão às aptidões mnémicas supostamente adquiridas pela mestra de obras para se lembrar dos diferentes provedores/terceiros e seus campos de intervenção, informação básica que deve introduzir em um sistema para que seja útil. Estes autores mostram, sobretudo a reconfiguração dos papéis supostos pelo trabalho. Mesmo assim, o sistema usado na construção para gerar e-mails automáticos para as hierarquias, estabelece novas formas de controle de qualidade e produtividade. Traçabilidade e controle fusionam-se. Os autores convocam Foucault para ilustrar esse risco.

o conjunto dos autores mostra de que modo estes sistemas, ao redesenhar os circuitos de trabalho, ao criar novas redes laborais, reconfiguram as regras e a divisão do trabalho e ao mesmo tempo desmaterializam (ou até mesmo 'desencorporam') o trabalho. Especificamente, todos apontam para um traço comum: a digitalização do trabalho é acompanhada do aumento explícito ou implícito das exigências de rentabilidade. Exigências que não podem ser cumpridas e que aceleram o ritmo de trabalho e a competição entre colegas. Pinheiro et al., Salini et al., e Leduc e Ponge ilustram o aumento de procedimentos que incluem os objetos digitais que freiam ou fazem obstáculo à fluidez dos processos de trabalho e impedem os trabalhadores não só de alcançar suas metas organizacionais, mas também que se configuam como restrições e cargas de trabalho adicionais. A aceleração do ritmo, a sobrecarga e a competição entre aqueles que deveriam cooperar opera em detrimento da qualidade do trabalho e do serviço oferecido, tanto em termos de produtividade como de ética.

3- Ao desenvolver sistemas artificialmente inteligentes, baseados em algoritmos e redes, que tratam e disseminam informações de forma autônoma, uma forma de 'proletarização do conhecimento' está sendo construída (Stiegler citado por Salini et al.). Aqueles que eram até agora os especialistas de seus próprios ofícios, perdem o poder de decisão e se tornam parte, ou componente de um novo sistema de trabalho realizado por esses novos objetos. Esses sistemas inteligentes também são baseados na noção de redes e aprendizagem. Muitos são baseados na apreensão, interpretação e extrapolação dos dados inseridos pelos mesmos utilizadores ou clientes. Novas formas de co-serviços são assim construídas. O cliente participa da atividade de construção do serviço, mas remove uma parte do controle do operador. Dessa forma, uma parte do trabalho é terceirizada (Leduc \& Ponge; Pinheiro et al.). Os operadores tornam-se, assim, um componente do sistema e, muitas vezes, são forçados a ser polivalentes para que o novo sistema de trabalho possa funcionar. Polivalência extrema dá a sensação de perda de perícia. Ao dissecar o trabalho dessa maneira, os operadores perdem suas referências, perdem o controle das informações, mas muitas vezes vêem novas formas de responsabilidades que não podem assumir. 'O poder de agir' está em risco em todos os casos.

O que chama a atenção à leitura desses estudos é que todos convergem para o desenvolvimento de estratégias para o trabalho com estes novos meios de comunicação. São estratégias principalmente de regulação ou de compensação muito exigentes para o trabalhador, em vez de novas marcações que possibilitariam uma apropriação. Percebese uma tendência de adaptação dos trabalhadores, seja individual ou coletivamente, 
para tentar responder às novas configurações de trabalho, mas novas práticas ainda não são visíveis. Para compreender a não-apropriação de certas ferramentas parece essencial determinar a profundidade da transformação que está acontecendo nas experiências de trabalho que perturbam o significado, o sentido, atribuído ao valor trabalho assim como da cooperação, não só entre os colegas, mas também com os beneficiários do serviço. Em relação a alguns trabalhadores cujas novas atividades são mediatizadas ou digitalizadas observa-se um conflito em relação a valores pessoais e coletivos.

24 A digitalização coloca mais do que nunca, no centro dos debates, o desvio entre o prescrito e o efetivo, o que é interpretado e o que é real. Os artefatos digitais cristalizam neles a fronteira material desse antagonismo fundamental da ergonomia da atividade, sendo a concretização de uma representação prescrita do trabalho (desenvolvida pelos concetores e gestores) e colocando em jogo, em seus usos, a própria essência do trabalho real para as pessoas.

\section{Reflexões sobre as intervenções nestas 'novas' condições de trabalho}

Com a digitalização, existem outros paradigmas que devemos convocar. Não se trata apenas de uma evolução da numerização, em andamento desde a introdução das TIC: com a digitalização está sendo definida uma nova configuração do trabalho real.

Do mesmo modo, por exemplo, as formas de implementação dessa digitalização representam novos problemas. Com exceção de Pinheiro et al., que focam seu estudo nas condições de implementação do programa de digitalização do Instituto Nacional do Seguro Social, é interessante notar que os demais autores apenas falam indiretamente sobre essas condições. Uma das explicações é a observação de que é necessário entender primeiro a digitalização e suas conseqüências para analisar as formas de implementá-la. Estaríamos então em um estágio de imaturidade digital que impede ter as ferramentas epistemológicas e teóricas adequadas.

Nas quatro contribuições, o fenômeno digital observado ocorre em um contexto de grandes empresas ou grandes instituições ou corporações. No plano de fundo estão os 'abandonados' pela onda digital: trabalhadores informais, precários, pobres, atividades com baixo nível de investimento. Nas escalas estudadas, um desenvolvimento digital é mostrado seguindo um caminho 'top down', transportado rapidamente, em diferentes áreas de organização do trabalho.

Em filigrana, duas estratégias diferentes de introdução e implementação são identificadas:

29 1) uma estratégia 'brutal', acompanhada de formação, que geralmente é realizada em paralelo com a introdução dos artefatos nas práticas de trabalho (Leduc \& Ponge), muitas vezes após a sua implementação.

2) uma estratégia de desenvolvimento evolutivo dos instrumentos digitais, tanto dos próprios suportes - transferindo suporte em tablets em smartphones (Chambonnière et al.) - quanto dos programas de computador (Salini et al.). ou das configurações organizacionais (Pinheiro et al.). 
31 Nenhuma dessas duas estratégias de implementação é adaptada de acordo com as conclusões dos autores. Seja porque as ações de formação são conduzidos sob uma abordagem funcional (ensino de novas funções com novos artefactos), ou porque a vontade de mudança gradual, ou desenvolvimento evolutivo, -muitas vezes sustentado por uma dinâmica de co-conceção traz consigo a necessidade de um ajuste permanente, um esforço para atualização constante das práticas que impedem os trabalhadores de estabilizem o seu contexto para que possam ser facilitadores ou atores de novas práticas.

32 Com esta conclusão, Salini et al., propõe novas abordagens de formação para responder aos desafios da digitalização nos serviços estudados. Eles propõem acompanhar os processos de apropriação com espaços concebidos como oportunidades de formação para troca de experiências. Proposta que é discutida e complementada com as de Pinheiro et al.

33 Leduc e Ponge e Chambonnière et al, insistem sobre a necessidade de rever os quadros analíticos, à luz de estudos de caso, de intervir preventivamente. Suas discussões e conclusões sugerem que se deve acompanhar o processo de digitalização do trabalho em diferentes níveis, a fim de preservar e otimizar as condições de trabalho. Seja intervindo com os atores da gestão ou com os concetores de novas tecnologias.

\section{Conclusão}

A digitalização não é um 'mal necessário'. É uma construção social e humana. Pode existir somente se a ancoragem social existir. $\mathrm{O}$ fenômeno tecnológico que vivemos mostra que uma parte da sociedade está pronta para receber a onda digital. No entanto, este desenvolvimento tecnológico não só acarreta o risco de uma fratura tecnológica ou social, mas desafia a própria noção de Trabalho para todos, para pesquisadores e profissionais do Trabalho. Os sistemas informáticos não trabalham, mas é o trabalho humano que evolui quando confrontado com essas novas tecnologias.

35 A evolução da digitalização põe em discussão os referenciais teóricos e metodológicos com os quais ela deve ser captada, observada e analisada. Ela levanta questões sobre as formas de assistência que podemos propor para reconhecer o trabalho real executado por indivíduos que estão empenhados em fazer o melhor possível nestas 'novas' condições de trabalho.

O que os quatro artigos que compõem este número temático nos mostra é que a onda está sendo vivida de maneira semelhante em diferentes contextos. Também nos mostra a necessidade de analisar essa evolução com abordagens abrangentes, analisando não apenas os artefatos digitais que a materializam, mas também as construções sociotécnicas que a materializam e nas quais se inscrevem. Ignorar as dimensões sociais e políticas em que esses desenvolvimentos se inscrevem significaria, em muitos casos, deixar de lado as razões que justificam sua implementação e as principais conseqüências negativas em termos de saúde para aqueles que são confrontados com certas situações de trabalho. Finalmente, eles nos ensinam que, como profissionais (cientistas ou especialistas) das condições de trabalho, é urgente que possamos intervir na construção desses artefactos para serem verdadeiros 'acompanhantes' das práticas de trabalho. 
Para os ergonomistas que trabalham com os concetores desses artefatos, sabemos que o maior risco está na representação e compreensão dos conceitos de trabalhadores e de trabalho. Além disso, muitas vezes pode-se ouvir discursos de gestores ou concetores que dizem 'recolocar o humano no centro das preocupações' ou realizar 'processos participativos' ou 'co-conceção'. No entanto, nestes discursos ou metodologias préfabricados, o 'Humano' considerado é uma figura cujas práticas são codificados para preservar ações úteis para a representação funcional do sistema de trabalho. É importante que possamos restabelecer a complexidade e a riqueza do trabalho humano em suas dimensões situada, social e incorporada, na concepção de novos espaços de trabalho digitais e acompanhar as diferentes formas que assume a digitalização para que se possa construir a partir do trabalho real e da experiência das pessoas. Assim, essas evoluções não estariam ao serviço da aceleração do ritmo, da sobrecarga de trabalho e da perda de sentido, mas a serviço da cooperação, da saúde e dos resultados das equipes de trabalho.

\section{BIBLIOGRAFIA}

Ayyagari, R., Grover, V., \& Purvis, R. (2011). Technostress: technological antecedents and implications. MIS quarterly, 35(4), 831-858.

Baudin, C. (2017a). La perte des sens et du sens des corps technicisés. Actes FISO, Festival International de Sociologie, La fabrication des corps au 21e siècle.15p.

Baudin, C. (2017b). Au coeur du sensible: l'or 'profane' de Madre de Dios. In s. d. Ph.Geslin (Ed.), L'anthropotechnologie - Cultures et Conception (Ed. ISTE, pp. 71-101). London.

Bobillier-Chaumon, M-E., \& Clot Y. (2016). Clinique de l'usage : Les artefacts technologiques comme développement de l'activité, Revue @ctivités, vol. 13, numéro 2, 8p.

Bobillier-Chaumon, M-E., (2017). Du rôle des TIC dans la transformation digitale de l'activité et de la santé au travail, ANACT, la Revue des Conditions de Travail, Sept, nº, 16-24.

De Stefano, V. (2015). The Rise of the Just-in-Time Workforce: On-Demand Work, Crowdwork, and Labor Protection in the Gig-Economy. Comparative Labor Law \& Policy Journal, 37, 471-503.

Degryse, C. (2016). Digitalisation of the economy and its impact on labour markets. Research paper, ETUI, 81p.

Degryse, C. (2017). Shaping the world of work in the digital economy. ETUI, 11p.

Drahokoupil, J., \& Fabo, B. (2016). The platform economy and the disruption of the employment relationship. ETUI, 6p.

Frey, C. B., \& Osborne, M. A. (2017). The future of employment: how susceptible are jobs to computerisation? Technological Forecasting and Social Change, 114(January), 254-280.

Latour, B. (1991). Nous n'avons jamais été modernes. Essai d'anthropologie symétrique, Paris: La Découverte. 
Loebbecke, C., \& Picot, A. (2015). Reflections on societal and business model transformation arising from digitization and big data analytics: A research agenda. The Journal of Strategic Information Systems, 24(3), 149-157.

Mathé, A. (2015) Numérique ou Digital. In https://www.blogdumoderateur.com/numerique-oudigital/ [consultado en 14.03.2018]

Mériau \& Rousseau (2017). Mieux travailler à l'ère du numérique: Définir les enjeux et soutenir l'action, ANACT, la Revue des Conditions de Travail, Sept, $\mathrm{n}^{\circ} 6$.

Murillo, D., Buckland, H., \& Val, E. (2017). When the sharing economy becomes neoliberalism on steroids: Unravelling the controversies. Technological Forecasting and Social Change, 125, 66-76. Organisation for Economic Co-operation and Development (OECD). (2017). OECD Digital Economy Outlook 2017. Paris: OECD.

Prassl, J., \& Risak, M. (2016). Uber, Taskrabbit, and Co.: Platforms as Employers-Rethinking the Legal Analysis of Crowdwork. Comparative Labor Law \& Policy Journal, 37(3), 604-619.

Rabardel, P. (2005). 13. Instrument, activité et développement du pouvoir d'agir. In Entre connaissance et organisation: l'activité collective (pp. 251-265). La Découverte.

Sadin, É. (2015). La vie algorithmique. Critique de la raison numérique. Paris: L'Échappée Shu, Q., Tu, Q., \& Wang, K. (2011). The impact of computer self-efficacy and technology dependence on computer-related technostress: A social cognitive theory perspective. International Journal of Human-Computer Interaction, 27(10), 923-939.

Valenduc, G., \& Vendramin, P. (2016). Work in the digital economy: sorting the old from the new. ETUI, 52p.

Valenduc, G., \& Vendramin, P. (2017). Digitalisation, between disruption and evolution. Transfer: European Review of Labour and Research, 23(2), 121-134.

Watson, R., \& Stafford, O. (2016). Shaping the new world of work. ETUI.

\section{NOTAS}

1. Várias iniciativas foram organizadas nos últimos anos. Para citar algumas realizadas em França ultimamente: a rede ANACT-ARACT França publicou na sua revista um número sobre a numerização no trabalho: 'Mieux travailler à l'ère du numérique: Definir les Enjeux et Soutenir les Actions'; Revue sur les conditions de travail, nº 6, Spt. 2017; O CNAM realizou no dia 10 de março de 2018, uma jornada de reflexão sobre trabalho e numérico; a segunda edição da universidade de verão multidisciplinar e internacional do trabalho de Bordeaux (França) foi realizada sobre o tema emprego e inovação tecnológica, entre 2 e 06 de julho de 2018. 


\section{AUTORES}

\section{CAROLE BAUDIN}

Laboratoire 'User centered Design'

Haute École Arc Ingénierie

University of Applied Sciences and Arts of Western Switzerland, HES SO, Espace de l'Europe 11, 2000 Neuchâtel Suisse

carole.baudin@he-arc.ch

\section{PATRICIO NUSSHOLD}

Laboratoire PCPP (Psychologie Clinique, Psychopathologie, Psychanalyse)

Université Paris Descartes - Sorbonne Paris Cité.

Institut de Psychodynamique du Travail - ASTI, 7 rue Clovis, 75005, Paris, France,

patricio.nusshold@ast-i.org 